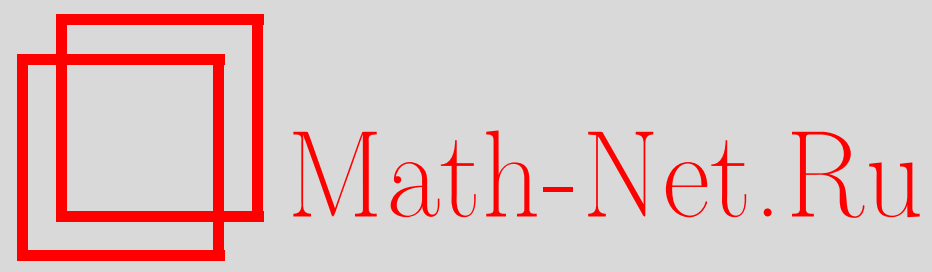

А. А. Владимиров, Оценки числа собственных значений самосопряженных оператор-функций, Матем. заметки, 2003, том 74, выпуск 6, 838-847

DOI: https://doi.org/10.4213/mzm321

Использование Общероссийского математического портала Math-Net.Ru подразумевает, что вы прочитали и согласны с пользовательским соглашением http://www.mathnet.ru/rus/agreement

Параметры загрузки:

IP : 3.89 .197 .203

26 апреля 2023 г., 16:49:55

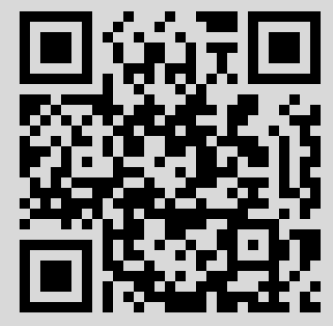




\section{ОЦЕНКИ ЧИСЛА СОБСТВЕННЫХ ЗНАЧЕНИЙ САМОСОПРЯЖЕННЫХ ОПЕРАТОР-ФУНКЦИЙ}

\section{А. А. Владимиров}

Рассматривается определенная на отрезке $[\sigma, \tau] \subset \mathbb{R}$ оператор-функция $F$, значениями которой являются полуограниченные самосопряженные операторы в гильбертовом пространстве $\mathfrak{H}$. С оператор-функцией $F$ связываются величины $\mathcal{N}_{F}$ и $\nu_{F}(\lambda)$, представляющие собой, соответственно, число собственньх значений оператор-функции $F$ на полуинтервале $[\sigma, \tau)$ и число отрицательных собственных значений оператора $F(\lambda)$ при произвольном $\lambda \in[\sigma, \tau]$. Устанавливаются условия, при которых справедлива оценка $\mathcal{N}_{F} \geqslant \nu_{F}(\tau)-\nu_{F}(\sigma)$. Также устанавливаются условия, при которых справедливо равенство $\mathcal{N}_{F}=\nu_{F}(\tau)-\nu_{F}(\sigma)$. Полученные результаты применяются к обыкновенным дифференциальным оператор-функциям на конечном отрезке.

Библиограбия: 7 названий.

\section{1. Общие утверждения}

Пусть $F$ есть определенная на отрезке $[\sigma, \tau] \subset \mathbb{R}$ оператор-функция, значениями которой являются самосопряженные операторы с компактной резольвентой в гильбертовом пространстве $\mathfrak{H}$. В дальнейшем будет предполагаться выполнение следующих двух условий:

(a) операторы $F(\lambda)$ равномерно по $\lambda \in[\sigma, \tau]$ ограничены снизу;

(б) оператор-функция $V$, удовлетворяющая тождеству

$$
V(\lambda) \equiv(F(\lambda)-i)^{-1}
$$

(здесь $i$ - мнимая единища), непрерьвна на отрезке $[\sigma, \tau]$ в смысле равномерной операторной топологии.

Через $\mathscr{N}_{F}$ будет в дальнейшем обозначаться сосчитанное с учетом кратности число собственных значений оператор-функции $F$, лежащих на полуинтервале $[\sigma, \tau)$. При этом, как обычно, под собственным значением кратности $m$ оператор-функции $F$ понимается число $\lambda \in[\sigma, \tau]$, для которого $\operatorname{dim} \operatorname{ker} F(\lambda)=m$.

Через $\nu_{F}(\lambda)$ будет в дальнейшем обозначаться сосчитанное с учетом кратности число отрицательных собственных значений оператора $F(\lambda)$.

Целью текущего раздела является изучение вопроса о связи между величинами $\nu_{F}(\lambda)$ и $\mathcal{N}_{F}$.

Работаподдержана Российским фондом фундаментальных исследований, гранты № 01-01-00691 и № 03-01-06426. 
1.1. Случай без дополнительных ограничений на оператор-функцию $F$. Справедливо следующее утверж дение.

ТеОрема 1. Пусть выполнены условия (а) и (б). Тогда имеет место неравенство

$$
\mathcal{N}_{F} \geqslant \nu_{F}(\tau)-\nu_{F}(\sigma) \text {. }
$$

Для доказательства теоремы 1 нам понадобится следующая вспомогательная последовательность числовых функций.

ОпРЕДЕЛЕниЕ 1. Через $\Lambda_{m}, m=1,2, \ldots$, будут обозначаться определенные на отрезке $[\sigma, \tau]$ числовые функции со следующим свойством: при любом $\lambda \in[\sigma, \tau]$ число $\Lambda_{m}(\lambda)$ есть $m$-ое снизу (с учетом кратности) собственное значение оператора $F(\lambda)$.

При выполнении условий (а) и (б) определение 1 корректно, поскольку тогда операторы $F(\lambda)$ ограничены снизу и имеют чисто дискретньй спектр.

Лемма 1. Пусть выполнены условия (а) и (б). Тогда кажсдая из функиий $\Lambda_{m}$ непрерывна на всем отрезке $[\sigma, \tau]$.

ДокАЗАТЕЛЬСтво. Зафиксируем вешественное число $C$, для которого оператор $F(\lambda)-C$ будет положителен независимо от выбора $\lambda \in[\sigma, \tau]$. Такое $C$ сушествует в силу предположения (a).

Обозначим через $\Theta_{m, C}$ определенные на отрезке $[\sigma, \tau]$ числовые функции, которые любому $\lambda \in[\sigma, \tau]$ ставят в соответствие $m$-ое сверху (с учетом кратности) собственное значение оператора $(F(\lambda)-C)^{-1}$. Из предположения (б) следует, что оператор-функция $(F-C)^{-1}$ непрерьвна на отрезке $[\sigma, \tau]$ в смысле равномерной операторной топологии. Поэтому числовые функции $\Theta_{m, C}$ непрерывны на отрезке $[\sigma, \tau]$ (см., например, теорему $[1$, с. 257$])$. Однако для выбранного числа $C$ справедливы тождества

$$
\Theta_{m, C}(\lambda) \equiv \frac{1}{\Lambda_{m}(\lambda)-C} .
$$

Следовательно, функции $\Lambda_{m}$ непрерьвны на отрезке $[\sigma, \tau]$. Тем самьм, лемма доказана.

ДоКАЗАТЕЛЬСТво ТЕОРЕМЫ 1. Непосредственно из определения величин $\nu_{F}(\lambda)$ и функций $\Lambda_{m}$ следует, что не менее $\nu_{F}(\tau)-\nu_{F}(\sigma)$ функций из последовательности $\Lambda_{m}$ принимают неотрицательное значение в точке $\sigma$ и отрицательное - в точке $\tau$. Из утверждаемой леммой 1 непрерывности функций $\Lambda_{m}$ потому следует, что не менее $\nu_{F}(\tau)-$ $\nu_{F}(\sigma)$ функций из последовательности $\Lambda_{m}$ имеют нуль на полуинтервале $[\sigma, \tau)$. Утверждение теоремы теперь немедленно получается из определения функций $\Lambda_{m}$.

1.2. Случай монотонности оператор-функции $F$. При формулировке следующих утверждений будет использоваться понятие замыкания квадратичной формы. Определение этого понятия можно найти в [2, гл. VI]. Кроме того, при формулировке следуюших утверждений будет предполагаться вьполнение такого условия:

(в) пусть определенная на отрезке $[\sigma, \tau]$ функция $\mathfrak{f}$, значениями которой являются квадратичные формы в пространстве $\mathfrak{H}$, такова, что при любом $\lambda \in[\sigma, \tau]$ квадратичная форма $\mathfrak{f}(\lambda)$ есть замыкание определенной на $\operatorname{dom} F(\lambda)$ квадратичной формы $\langle F(\lambda) \cdot, \cdot\rangle_{\mathfrak{H}} ;$ тогда область определения квадратичной формы $\mathfrak{f}(\lambda)$ не зависит от выбора значения $\lambda \in[\sigma, \tau]$. 
Ниже не зависящая от выбора $\lambda \in[\sigma, \tau]$ область определения квадратичной формы $\mathfrak{f}(\lambda)$ будет обозначаться через dom $\mathfrak{f}$.

ТЕОРема 2. Пусть выполнены условия (а)-(в). Пусть также при любых $\lambda_{1}, \lambda_{2} \in[\sigma, \tau]$, удовлетворяющ,х неравенству $\lambda_{1}<\lambda_{2}$, определенная на линейном подпространстве $\operatorname{dom} \mathfrak{f}$ квадратичная форма

$$
\mathfrak{f}\left(\lambda_{1}\right)-\mathfrak{f}\left(\lambda_{2}\right)
$$

является полохстельной. Тогда имеет место равенство

$$
\mathcal{N}_{F}=\nu_{F}(\tau)-\nu_{F}(\sigma)
$$

Доказательство теоремы 2 опирается на следующее приводимое без доказательства утверждение, представляющее собой простое следствие известного вариационного принципа $\left[3\right.$, гл. 1 , теорема $\left.13^{\text {bis }}\right]$.

УТВЕРЖДЕНИЕ 1. Пусть Т есть ограниченный снизу самосопряэсенный оператор в гильбертовом пространстве $\mathfrak{H}$, спектр которого чисто дискретен на луче $(-\infty, 0)$. Пусть $\mathfrak{t}$ есть замыкание определенной на линейном подпространстве dom $\mathbf{T}$ квадратичной формы $\langle\mathbf{T} \cdot, \cdot\rangle_{\mathfrak{H}}$. Тогда число отрицательных собственных значений оператора Т равно максимуму размерностей подпространств $\mathfrak{M} \subset \operatorname{dom} \mathfrak{t}$, на которых квадратичная форма $\mathfrak{t}$ отрииательна.

ДоКАЗАТЕЛЬСТво ТЕОРЕмЫ 2. Зафиксируем произвольное число $m \in \mathbb{N}$, а также произвольные числа $\lambda_{1}, \lambda_{2} \in[\sigma, \tau]$, удовлетворяющие неравенству $\lambda_{1}<\lambda_{2}$. Очевидно, что найдется $m$-мерное подпространство $\mathfrak{M} \subset \operatorname{dom} F\left(\lambda_{1}\right)$, на котором квадратичная форма оператора $F\left(\lambda_{1}\right)-\Lambda_{m}\left(\lambda_{1}\right)$ будет неположительна. Однако при выполнении условий теоремы указанное подпространство $\mathfrak{M}$ будет подпространством области определения квадратичной формы $\mathfrak{f}\left(\lambda_{2}\right)$, причем квадратичная форма

$$
\mathfrak{f}\left(\lambda_{2}\right)-\Lambda_{m}\left(\lambda_{1}\right)\|\cdot\|_{\mathfrak{H}}^{2}
$$

будет на подпространстве $\mathfrak{M}$ отрицательна. Из утверждения 1 теперь немедленно вытекает справедливость неравенства $\Lambda_{m}\left(\lambda_{2}\right)<\Lambda_{m}\left(\lambda_{1}\right)$.

Ввиду произвольности выбора чисел $m, \lambda_{1}$ и $\lambda_{2}$, сказанное означает, что каждая из функций $\Lambda_{m}$ строго убьвает на отрезке $[\sigma, \tau]$.

Однако из непрерывности и строгого убывания функций $\Lambda_{m}$ следует, что величина $\mathscr{N}_{F}$ в точности равна числу таких функций из последовательности $\Lambda_{m}$, которые принимают неотрицательное значение в точке $\sigma$ и отрицательное - в точке $\tau$. Поскольку число таких функций есть $\nu_{F}(\tau)-\nu_{F}(\sigma)$, то утверждение теоремы справедливо.

1.3. Случай отрицательности типа собственных значений оператор-функции $F$. Содержание следующего утверждения тесно связано с известным в теории операторных пучков понятием типа собственного значения. 
ТЕОрема 3. Пусть выполнены условия (а)-(в). Пусть также некоторая определенная на отрезке $[\sigma, \tau]$ функиия $\mathfrak{f}^{\prime}$, значениями которой являются квадратичные формы в пространстве $\mathfrak{H , ~ о б л а д а е т ~ с л е д у ю щ и м и ~ с в о и ̆ с т в а м и : ~}$

1) при любом $\lambda \in[\sigma, \tau]$ областью определения квадратичной формы $\mathfrak{f}^{\prime}(\lambda)$ является линейное подпространство dom f;

2) квадратичные формы $\mathfrak{f}^{\prime}(\lambda)$ равномерно по $\lambda \in[\sigma, \tau]$ ограничены относительно произвольной нормы $\|\cdot\|_{\mathfrak{F}}$, превращающей $\operatorname{dom} \mathfrak{f}$ в банахово пространство;

3) при любом $y \in \operatorname{dom} \mathfrak{f}$ числовая функиия $\Phi_{y}$, удовлетворяющая на отрезке $[\sigma, \tau]$ тождеству

$$
\Phi_{y}(\lambda) \equiv \mathfrak{f}(\lambda)[y]
$$

является непрерывно дифференцируемой и удовлетворяет тождеству

$$
\Phi_{y}^{\prime}(\lambda) \equiv \mathfrak{f}^{\prime}(\lambda)[y]
$$

4) для любой собственной пары $\{\lambda, y\}$ оператор-функиии $F$ справедливо неравенство $\mathfrak{f}^{\prime}(\lambda)[y]<0$.

Тогда имеет место равенство

$$
\mathcal{N}_{F}=\nu_{F}(\tau)-\nu_{F}(\sigma)
$$

ДокАЗАТЕЛЬСТво. Шаг 1. Пусть $m \in \mathbb{N}$ и $\lambda_{0} \in[\sigma, \tau]$ - произвольные числа, удовлетворяющие равенству $\Lambda_{m}\left(\lambda_{0}\right)=0$.

В силу предположения (а) найдется вещественное число $C$, для которого квадратичная форма $\mathfrak{f}\left(\lambda_{0}\right)-C\|\cdot\|_{\mathfrak{H}}^{2}$ мажорирует квадратичную форму $\|\cdot\|_{\mathfrak{H}}^{2}$. Нетрудно убедиться, что для такого $C$ линейное пространство $\operatorname{dom} \mathfrak{f}$ полно относительно нормы

$$
\mid y \|_{\mathfrak{F}}:=\sqrt{\mathfrak{f}\left(\lambda_{0}\right)[y]-C \mid y \|_{\mathfrak{H}}^{2}}
$$

В дальнейшем гильбертово пространство, заданное на $\operatorname{dom} \mathfrak{f}$ нормой (1), будет обозначаться через $\mathfrak{F}$.

Заметим, что при любом $\lambda \in[\sigma, \tau]$ квадратичная форма $\mathfrak{f}(\lambda)$ ограничена относительно нормы $\|\cdot\|_{\mathfrak{F}}$. Действительно, поскольку норма $\|\cdot\|_{\mathfrak{H}}$ мажорируется нормой $\|\cdot\|_{\mathfrak{F}}$, то из полуограниченности квадратичной формы $\mathfrak{f}(\lambda)$ относительно нормы $\|\cdot\|_{\mathfrak{H}}$ следует, что квадратичная форма $\mathfrak{f}(\lambda)$ полуограничена относительно нормы $\|\cdot\|_{\mathfrak{F}}$. Однако ограниченность всюду определенной полуограниченной квадратичной формы есть несложное следствие теоремы о замкнутом графике (см., например, [2, гл. III, теорема 5.20]).

Сказанное означает, что тождество

$$
\langle(1+K(\lambda)) y, y\rangle_{\mathfrak{F}} \equiv \mathfrak{f}(\lambda)[y]
$$

определяет оператор-функцию $K$, значениями которой являются ограниченные самосопряженные операторы в пространстве $\mathfrak{F}$. При этом, как следует из компактности резольвенты оператора $F\left(\lambda_{0}\right)$, оператор $K\left(\lambda_{0}\right)$ является компактным. Из свойств функции $\mathfrak{f}^{\prime}$ следует также, что оператор-функция $K$ является дифференцируемой в смысле 
равномерной операторной топологии, причем ее производная $K^{\prime}$ удовлетворяет тождеству

$$
\left\langle K^{\prime}(\lambda) y, y\right\rangle_{\mathfrak{F}} \equiv \mathfrak{f}^{\prime}(\lambda)[y],
$$

и операторы $K^{\prime}(\lambda)$ равномерно по $\lambda \in[\sigma, \tau]$ ограничены.

Шаг 2. Разложим пространство $\mathfrak{F}$ в прямую сумму

$$
\mathfrak{F}_{-} \oplus \mathfrak{F}_{0} \oplus \mathfrak{F}_{+}
$$

где

$\mathfrak{F}$ - есть инвариантное подпространство оператора $K\left(\lambda_{0}\right)$, отвечающее той части его спектра, которая лежит на луче $(-\infty,-1)$;

$\mathfrak{F}_{0}$ есть инвариантное подпространство оператора $K\left(\lambda_{0}\right)$, отвечающее собственному значению -1 ;

$\mathfrak{F}_{+}$есть инвариантное подпространство оператора $K\left(\lambda_{0}\right)$, отвечающее той части его спектра, которая лежит на луче $(-1,+\infty)$.

Из компактности оператора $K\left(\lambda_{0}\right)$ следует существование такого $\varepsilon>0$, для которого на подпространстве $\mathfrak{F}$ - справедливы оценки

$$
\left\langle K\left(\lambda_{0}\right) y, y\right\rangle_{\mathfrak{F}} \leqslant(-1-\varepsilon)\|y\|_{\mathfrak{F}}^{2},
$$

а на подпространстве $\mathfrak{F}+$ справедливы оценки

$$
\left\langle K\left(\lambda_{0}\right) y, y\right\rangle_{\mathfrak{F}} \geqslant(-1+\varepsilon)\|y\|_{\mathfrak{F}}^{2} .
$$

Однако из условий теоремы и из первой теоремы о представлении (см. [2, гл. VI, теорема 2.1]) следует, что для любого ненулевого вектора $y \in \mathfrak{F}_{0}$ вьполняется неравенство

$$
\left\langle K^{\prime}\left(\lambda_{0}\right) y, y\right\rangle_{\mathfrak{F}}<0
$$

Поэтому из оценки (2) и конечномерности подпространства $\mathfrak{F}$ - следует, что найдется правая проколотая полуокрестность $\mathscr{U}^{+}\left(\lambda_{0}\right)$ точки $\lambda_{0}$ такая, что для любого $\lambda \in$ $\mathscr{U}^{+}\left(\lambda_{0}\right) \cap[\sigma, \tau]$ квадратичная форма оператора $1+K(\lambda)$ будет отрицательна на подпространстве $\mathfrak{F}_{-} \oplus \mathfrak{F}_{0}$. Аналогично, из оценки (3) и равномерной ограниченности операторов $K^{\prime}(\lambda)$ следует, что найдется левая проколотая полуокрестность $\mathscr{U}^{-}\left(\lambda_{0}\right)$ точки $\lambda_{0}$ такая, что для любого $\lambda \in \mathscr{U}^{-}\left(\lambda_{0}\right) \cap[\sigma, \tau]$ квадратичная форма оператора $1+K(\lambda)$ будет положительна на подпространстве $\mathfrak{F}_{0} \oplus \mathfrak{F}_{+} \cdot$

Заметим теперь, что подпространство $\mathfrak{F}-\oplus \mathfrak{F}_{0}$ имеет размерность $\geqslant m$. Поэтому из определения оператор-функции $K$ и утверждения 1 следует, что при любом

$$
\lambda \in \mathscr{U}^{+}\left(\lambda_{0}\right) \cap[\sigma, \tau]
$$

справедливо неравенство $\Lambda_{m}(\lambda)<0$. Аналогично, подпространство $\mathfrak{F}_{0} \oplus \mathfrak{F}_{+}$имеет коразмерность $<m$. Поэтому при любом

$$
\lambda \in \mathscr{U}^{-}\left(\lambda_{0}\right) \cap[\sigma, \tau]
$$

справедливо неравенство $\Lambda_{m}(\lambda) \geqslant 0$. 
Шаг 3. Результаты предыдущего шага можно резюмировать следующим образом: каковы бы ни были $m \in \mathbb{N} u \lambda \in[\sigma, \tau]$, удовлетворяющие равенству $\Lambda_{m}(\lambda)=0$, функиия $\Lambda_{m}$ неотрицательна в некоторой левой полуокрестности точки $\lambda$ и отрицательна в некоторой правой проколотой полуокрестности точки $\lambda$. Поскольку любая из функций $\Lambda_{m}$ непрерывна в силу леммы 1 , то эти результаты означают, что любая из функций $\Lambda_{m}$ строго убывает в каждом из своих нулей. Отсюда, в свою очередь, следует, что каждая из функций $\Lambda_{m}$ имеет не более одного нуля, слева от которого она принимает только положительные, а справа - только отрицательные значения.

Повторяя теперь рассуждения, завершающие доказательство теоремы 2 , убеждаемся в справедливости утверждения доказываемой теоремы.

\section{2. Применение к дифференциальным оператор-функциям}

Рассмотрим теперь определенную на отрезке $[\sigma, \tau] \subset \mathbb{R}$ оператор-функцию $S$, значениями которой являются самосопряженные операторы в пространстве $L_{2}[a, b]$, заданные дифференциальным выражением

$$
\sum_{k=0}^{n}(-1)^{n-k}\left(p_{k}(x, \lambda) y^{(n-k)}(x)\right)^{(n-k)}
$$

и краевыми условиями

$$
(U(\lambda)-1) y^{\vee}+i(U(\lambda)+1) y^{\wedge}=0 .
$$

Здесь и далее предполагается вьполнение следующих условий:

(1) вещественные функции $p_{k}, k=0, \ldots, n$, непрерьвны на прямоугольнике $[a, b] \times$ $[\sigma, \tau]$

(2) функция $p_{0}$ положительна на прямоугольнике $[a, b] \times[\sigma, \tau]$;

(3) при любом фиксированном $\lambda \in[\sigma, \tau]$ справедливы включения

$$
p_{k}(\cdot, \lambda) \in C^{n-k}[a, b], \quad k=0, \ldots, n ;
$$

(4) матрица-функция $U$, значениями которой являются унитарные комплексныематрицы порядка $2 n$, непрерьвна на отрезке $[\sigma, \tau]$.

Через $y^{\wedge}$ и $y^{\vee}$ в (5) обозначены векторы

$$
y^{\wedge}:=\left(\begin{array}{c}
y(a) \\
y^{\prime}(a) \\
\ldots \ldots \ldots \\
y^{(n-1)}(a) \\
y(b) \\
y^{\prime}(b) \\
\ldots \ldots \ldots \\
y^{(n-1)}(b)
\end{array}\right), \quad y^{\vee}:=\left(\begin{array}{c}
y^{[2 n-1]}(a) \\
y^{[2 n-2]}(a) \\
\ldots \ldots \ldots \ldots \\
y^{[n]}(a) \\
-y^{[2 n-1]}(b) \\
-y^{[2 n-2]}(b) \\
\ldots \ldots \ldots \ldots \\
-y^{[n]}(b)
\end{array}\right)
$$

(cp. с $[4$, формула $(7.50)])$. Здесь $y^{[n+m]}(x)$ суть обычные сокращения

$$
y^{[n+m]}(x):=\sum_{k=0}^{m}(-1)^{m-k}\left(p_{k}(x, \lambda) y^{(n-k)}(x)\right)^{(m-k)}, \quad m=0, \ldots, n
$$


(ср. с $[5, \S 15$, формула (3)] и [4, формула (7.46)]).

Из теоремы $[4$, теорема 7.5$]$ следует, что значениями оператор-функции $S$, заданной посредством (4), (5), являются самосопряженные операторы.

2.1. Свойства оператор-функции $S$. Заданная посредством (4), (5) операторфункция $S$ обладает следующими свойствами.

Лемма 2. Пусть А есть матрица-функиия, удовлетворяющая тождеству

$$
A(\lambda) \equiv \frac{1}{2 \pi} \int_{\Gamma(\lambda)} \frac{z+1}{z-1} \cdot(U(\lambda)-z)^{-1} d z
$$

где U есть матрица-функция из краевых условий (5), а Г( $\lambda$ ) есть произвольный положительно ориентированный контур, внутренность которого не содержит точку 1 , но содержит все отличные от 1 собственные значения матрицы $U(\lambda)$.

Тогда при любом $\lambda \in[\sigma, \tau]$ для любой функиии $y \in \operatorname{dom} S(\lambda)$ справедливо равенство

$$
\langle S(\lambda) y, y\rangle_{L_{2}[a, b]}=\sum_{k=0}^{n} \int_{a}^{b} p_{k}(x, \lambda)\left|y^{(n-k)}(x)\right|^{2} d x+\left\langle A(\lambda) y^{\wedge}, y^{\wedge}\right\rangle_{\mathbb{C}^{2 n}}
$$

Лемма 3. Если матрица-функиия $U$ такова, что числовая функция $\operatorname{rank}(U-1)$ постоянна на отрезке $[\sigma, \tau]$, то операторы $S(\lambda)$ равномерно по $\lambda \in[\sigma, \tau]$ ограничены снизу.

Лемма 4. Оператор-функиия $V$, удовлетворяющая тождеству

$$
V(\lambda) \equiv(S(\lambda)-i)^{-1}
$$

непрерывна на отрезке $[\sigma, \tau]$ в смысле равномерной операторной топологии.

Лемма 5. При любом $\lambda \in[\sigma, \tau]$ замыканием определенной на линейном подпространстве $\operatorname{dom} S(\lambda)$ квадратичной формы $\langle S(\lambda) \cdot, \cdot\rangle_{L_{2}[a, b]}$ является квадратичная форма $\mathfrak{s}(\lambda)$ с областью определения

$$
\left\{y \in L_{2}[a, b] \mid y \in W_{2}^{n}[a, b], y^{\wedge} \perp \operatorname{ker}(U(\lambda)-1)\right\},
$$

удовлетворяющая тождеству

$$
\mathfrak{s}(\lambda)[y] \equiv \sum_{k=0}^{n} \int_{a}^{b} p_{k}(x, \lambda)\left|y^{(n-k)}(x)\right|^{2} d x+\left\langle A(\lambda) y^{\wedge}, y^{\wedge}\right\rangle_{\mathbb{C}^{2 n}}
$$


ДоКАЗАТЕЛЬСТВО ЛЕМмы 2. При помощи интегрирования по частям нетрудно установить справедливость тождества

$$
\langle S(\lambda) y, y\rangle_{L_{2}[a, b]} \equiv \sum_{k=0}^{n} \int_{a}^{b} p_{k}(x, \lambda)\left|y^{(n-k)}(x)\right|^{2} d x+\left\langle y^{\vee}, y^{\wedge}\right\rangle_{\mathbb{C}^{2 n}}
$$

Поэтому лемма будет доказана, если будет установлено, что для любых векторов $Y, Z \in$ $\mathbb{C}^{2 n}$, удовлетворяющих условию

$$
(U(\lambda)-1) Z+i(U(\lambda)+1) Y=0
$$

выполняется равенство

$$
\langle Z, Y\rangle_{\mathbb{C}^{2 n}}=\langle A(\lambda) Y, Y\rangle_{\mathbb{C}^{2 n}}
$$

Заметим, что условие (8) можно переписать в параметрической форме

$$
\begin{aligned}
Y & =(U(\lambda)-1) X, \\
Z & =-i(U(\lambda)+1) X, \\
X & \in \mathbb{C}^{2 n}
\end{aligned}
$$

При этом левая часть предполагаемого равенства (9) оказывается равна скалярному произведению

$$
\langle B(\lambda) X, X\rangle_{\mathbb{C}^{2 n}}
$$

где через $B(\lambda)$ обозначена матрица

$$
B(\lambda):=-i\left(U^{-1}(\lambda)-1\right) \cdot(U(\lambda)+1)=\frac{1}{2 \pi} \int_{\Gamma_{1}}\left(z^{-1}-1\right) \cdot(z+1) \cdot(U(\lambda)-z)^{-1} d z .
$$

Здесь $\Gamma_{1}$ есть положительно ориентированная граница кольца

$$
\{z \in \mathbb{C}|| z \mid \in(1-\varepsilon, 1+\varepsilon)\}, \quad \varepsilon<1 \text {. }
$$

С другой стороны, правая часть предполагаемого равенства (9) оказьвается равна скалярному произведению

$$
\left\langle B^{+}(\lambda) X, X\right\rangle_{\mathbb{C}^{2 n}}
$$

где через $B^{+}(\lambda)$ обозначена матрица

$$
B^{+}(\lambda):=\frac{1}{2 \pi} \int_{\Gamma(\lambda)}\left(z^{-1}-1\right) \cdot(z+1) \cdot(U(\lambda)-z)^{-1} d z
$$

Контур $\Gamma(\lambda)$ определен в формулировке доказьваемой леммы.

Между тем, функция $-i\left(z^{-1}-1\right)(z+1)$ обращается в нуль в точке $z=1$, а матрища $U(\lambda)$ заведомо не имеет присоединенных векторов, отвечающих собственному значению 1. Поэтому матрицы $B(\lambda)$ и $B^{+}(\lambda)$ равны. Следовательно, при вьполнении условия $(8)$ равенство (9) действительно справедливо. Тем самым лемма доказана. 
ДОКАЗАТЕЛЬСТВО ЛЕММЫ 3. При вьполнении условия $\operatorname{rank}(U(\lambda)-1) \equiv$ const матрица-функция $A$ является непрерывной на отрезке $[\sigma, \tau]$. Поэтому найдется такое вещественное число $C$, что определенная в пространстве $W_{2}^{n}[a, b]$ ограниченная квадратичная форма

$$
\mathfrak{s}_{C}(\lambda)[y] \equiv \sum_{k=0}^{n} \int_{a}^{b} p_{k}(x, \lambda)\left|y^{(n-k)}(x)\right|^{2} d x-\int_{a}^{b} C \cdot|y(x)|^{2} d x+\left\langle A(\lambda) y^{\wedge}, y^{\wedge}\right\rangle_{\mathbb{C}^{2 n}}
$$

будет неотрицательна независимо от выбора $\lambda \in[\sigma, \tau]$.

Однако из леммы 2 с очевидностью следует, что неотрищательность квадратичной формы $\mathfrak{s}_{C}(\lambda)$ влечет за собой неотрицательность оператора $S(\lambda)-C$ (обратное, вообще говоря, неверно). Значит, ранее определенное число $C$ представляет собой равномерную по $\lambda \in[\sigma, \tau]$ миноранту операторов $S(\lambda)$. Тем самьм утверждение леммы справедливо.

ДокАЗАтЕЛЬСтво ЛЕммы 4. Рассмотрим определенную на параллелепипеде $[a, b] \times$ $[a, b] \times[\sigma, \tau]$ функцию $G$, для которой при любом $\lambda \in[\sigma, \tau]$ функция $G(\cdot, \cdot, \lambda)$ представляет собой функцию Грина оператора $S(\lambda)+i$. Нетрудно проверить, что непрерывность функций $p_{k}$ и $U$ приводит к тому, что функция $G$ непрерывна на параллелепипеде $[a, b] \times[a, b] \times[\sigma, \tau]$. Отсюда с очевидностью вытекает справедливость утверждения леммы.

ДокАЗАТЕЛЬСТво лЕммы 5. Справедливость утверждения доказьваемой леммы нетрудно вывести из леммы 2 и возможности записи условия (8) в форме (10).

2.2. Оценки числа собственных значений. Из лемм $2-5$ и теорем $1-3$ вытекает справедливость следующих утверждений.

ТЕОРема 4. Пусть матрица-функиия $U$ такова, что числовая функция $\operatorname{rank}(U-1)$ постоянна на отрезке $[\sigma, \tau]$. Тогда имеет место оценка

$$
\mathcal{N}_{S} \geqslant \nu_{S}(\tau)-\nu_{S}(\sigma)
$$

ТЕОРЕМА 5. Пусть выполнены следующие условия:

1) подпространство $\operatorname{ker}(U(\lambda)-1)$ не зависит от выбора значения $\lambda \in[\sigma, \tau]$;

2) при любом фиксированном $x \in[a, b]$ функиии $p_{k}(x, \cdot), k=0, \ldots, n-1$, невозрастают; кроме того, при любом фиксированном $x \in[a, b]$ функиия $p_{n}(x, \cdot)$ строго убивает;

3) отвечающая матрице-функиии $U$ матрица-функиия $A$ (см. лемму 2) невозрастает в том смысле, что при любых $\lambda_{1}, \lambda_{2} \in[\sigma, \tau]$, удовлетворяюших неравенству $\lambda_{1}<\lambda_{2}$, самосопряженная матрица $A\left(\lambda_{1}\right)-A\left(\lambda_{2}\right)$ является неотрииательной.

Тогда имеет место равенство

$$
\mathscr{N}_{S}=\nu_{S}(\tau)-\nu_{S}(\sigma)
$$


ТЕОРемА 6. Пусть выполнены следующие условия:

1) подпространство $\operatorname{ker}(U(\lambda)-1)$ не зависит от выбора значения $\lambda \in[\sigma, \tau]$;

2) функции $p_{k}, k=0, \ldots, n$, обладают непрерывными на прямоугольнике $[a, b] \times$ $[\sigma, \tau]$ частными производными по второму аргументу;

3) матрица-функиия $U$ является непрерывно дифферениируемой;

4) для любой собственной пары $\{\lambda, y\}$ оператор-функиии $S$ имеет место неравенство

$$
\left.\sum_{k=0}^{n} \int_{a}^{b} \frac{\partial p_{k}(x, \mu)}{\partial \mu}\right|_{\mu=\lambda} \cdot\left|y^{(n-k)}(x)\right| d x+\left\langle A^{\prime}(\lambda) y^{\wedge}, y^{\wedge}\right\rangle_{\mathbb{C}^{2 n}}<0
$$

Тогда имеет место равенство

$$
\mathscr{N}_{S}=\nu_{S}(\tau)-\nu_{S}(\sigma)
$$

Отметим, что фигурирующее в формулировке теоремы 4 требование постоянности числовой функции $\operatorname{rank}(U-1)$ является существенным. Действительно, определенная на отрезке $[-1,1]$ оператор-функция $\widetilde{S}$, значениями которой являются операторы в $L_{2}[0, \pi]$ вида

$$
-y^{\prime \prime}, \quad y(0)=y(\pi)-\lambda y^{\prime}(\pi)=0
$$

(см. [2, гл. V, пример 4.14]), не удовлетворяет этому требованию, и для нее имеет место неравенство

$$
0=\mathscr{N}_{\widetilde{S}}<\nu_{\widetilde{S}}(1)-\nu_{\widetilde{S}}(-1)=1-0=1
$$

В качестве следствий теорем 4-6 могут быть получены некоторые известные оценки числа собственных значений дифференциальных оператор-функций-в частности, оценки, связанные с результатами работ [6] и [7].

\section{СПИСОК ЦИТИРОВАННОЙ ЛИТЕРАТУРЫ}

[1] Рисс Ф., Сёкефальви-Надь Б. Лекции по функциональному анализу. М.: Мир, 1979.

[2] Като Т. Теория возмущений линейных операторов. М.: Мир, 1972.

[3] Глазман И. М. Прямые методы качественного спектрального анализа сингулярных дифференциальных операторов. М.: Физматгиз, 1963.

[4] Рофе-Бекетов Ф. С., Холькин А. М. Спектральный анализ дифференциальных операторов. Связь спектральных и осцилляционных свойств. Мариуполь, 2001.

[5] Наймарк М. А. Линейные дифференциальные операторы. М.: Наука, 1969.

[6] Kamke E. Neue Herleitung der Oszillationssätze für die linearen selbstadjungierten Randwertaufgaben zweiter Ordnung // Math. Z. 1938. V. 44. P. 635-658.

[7] Mennicken R., Schmid H., Shkalikov A. A. On the eigenvalue accumulation of Sturm-Liouville problems depending nonlinearly on the spectral parameter // Math. Nachr. 1998. V. 189. P. $157-170$. 